\title{
Characterising powder flow properties - the need for a multivariate approach
}

\author{
Tim Freeman ${ }^{l *}$, Katrina Brockbank $^{l}$ and Jerome Sabathier ${ }^{2}$ \\ ${ }^{1}$ Freeman Technology Ltd, Tewkesbury, UK \\ ${ }^{2}$ ForS Instruments, Bailleau Pine, France
}

\begin{abstract}
Despite their widespread and well-established use, powders are challenging materials to work with, as evidenced by the common problems encountered during storage and processing, as well as in the quality and consistency of final products. The diverse range of unit operations used to handle and manipulate powders subject them to extremes of stress and flow regimes; from the high stress, static conditions present in hoppers to the dispersed, dynamic state of a fluidised bed dryer. It is therefore possible for a powder to behave a certain way in a given unit operation, but entirely differently in another. Many existing powder testing techniques don't deliver the required information as the test conditions do not represent the conditions in the process. Modern powder rheometers generate process relevant data by accurately measuring dynamic flow, bulk and shear properties. This approach enables a powder's response to aeration, consolidation, forced flow and changes in flow rate to be reliably quantified thereby simulating the conditions which a powder will be subjected to in process. This paper provides an introduction to powder rheology, including a comparison with traditional techniques, and uses case studies to demonstrate how powder rheology can be applied to optimise production processes and enhance product quality
\end{abstract}

\section{Introduction}

Powder and granular systems must be considered as a multiphase continuum whose behaviour is a function of both the fundamental properties of the powder and individual particles (for example, size, shape, surface texture and moisture content), and external factors such as humidity, temperature and consolidation history. As such powders and granular materials exhibit diverse and complex behaviour

Whilst manufacturers may be able to control some fundamental properties, such as particle size and crystal habit, most fundamental properties cannot not be dictated, especially for natural and biological materials. External factors such as temperature and humidly can also be regulated however this can often be extremely expensive and may only cover part of the lifecycle of the powder. As such powder behaviour is difficult to both predict and control. The manufacturer and end user must use a proactive approach in understanding the behaviour of a given powder or granular system through accurate and repeatable quantification of the powder properties, in particular the flowability. This is further complicated by the dependence of the flow behaviour on the stress and strain rate, meaning the test conditions should ideally be representative of the process conditions to which the powder is subjected

A number of well documented, traditional methods such as Angle of Repose (AOR), Flow meters (such as Critical Orifice Diameter) and Tapped Density tests (Carr's Index / Hauser Ratio) are widely available. Whilst these methods are typically cheap, quick and simple to use they are highly operator dependent, poorly repeatable and not overly differentiating [1]. Another widely accepted methodology is Shear Cell analysis. Whilst the mathematical principles are clearly defined, this method was originally designed to determine the incipient flow of continuous, cohesive powders at higher stress levels whereas as many processes and unit operations occur at low to moderate stresses. One major advantage of Shear Cell analysis is its use in hopper design,

Arguably, there are two major disadvantages with these methodologies. Firstly, these methods cannot characterise the full range of powder flowability/ cohesion. AOR and Flow Meters require the powder or granular material to flow through a funnel and are therefore unsuitable for cohesive powders. Tapped Density tests are also unsuitable for highly cohesive materials as the tapping force is insufficient to overcome the strong interparticular cohesive bonds meaning that the powder bed will not necessarily repack / consolidate when

\footnotetext{
* Corresponding author: tim.freeman@freemantech.co.uk
} 
tapped. A further issue arises with this test method when dealing with granular materials, as the heavier / denser particles are more likely to pack efficiently under their own mass, resulting in falsely low results.

Conversely Shear Cell analysis is typically less differentiating with respect to free-flowing powders and may not detect minor variations in the powder properties which impact on the process performance. Furthermore, due to particle size limitations, commercially available instruments are not readily available for larger granular materials. The second major disadvantage is that these methods is that they are not generally representative of the range of process conditions to which powders and grains are subjected during processing, indeed there is no standard test for measuring powder flow in all four flow regimes (plastic, inertial, fluidised and entrained flow) [2].

More recent development of powder rheometers has overcome these limitations, with these methods becoming more widely recognised and accepted, as demonstrated by the large number of journal articles available [2, 3]. Whilst this technology was primary developed to measure powders under dynamic conditions, the modular design and adaptability means that both the bulk (Compressibility and Permeability) and shear (Shear Cell and Wall Friction) properties are also easily measured. As such this technique is capable of evaluating both freeflowing and highly cohesive materials, even at larger particular sizes, not only allowing direct comparison of different materials (i.e. different raw ingredients) but also meaning that a single instrument can be used to monitor the process train from raw materials through to the finished product.

\section{Dynamic principle}

The rheological properties of the powder or granular systems are quantified through measurements of the resistance to flow. The FT4 Powder Rheometer (Freeman Technology, UK) reports this as a flow energy. This is obtained by means of a patented measurement principle that evaluates the resistance to the motion of a twisted blade passing along a prescribed path through a precise volume of the powder. The torque and force required to maintain movement of the blade are recorded and converted into a flow energy (figure 1). This principle has recently been modelled using DEM with the results of the computer model shown to be comparable to experimental values. [4]. Modification of the tip speed and helix angle also allow the stress and strain rates to be easily altered whilst different vessel and blade sizes allow the characterisation of powders with particle sizes ranging from a few nanometres to several millimetres.

Unlike traditional methodologies, dynamic test methods also incorporate sample conditioning to remove operator variability and consolidation history. This is achieved through the displacement of the whole sample to loosen and slightly aerate the powder with the aim of producing a standardised packing state. For ease of use the conditioning cycle(s) is normally incorporated into the test program however where consolidation/storage history is being investigated the sample can be pre -conditioned before storing the sample. Further improvements to repeatability are achieved by use of a vessel design which incorporates a splitting mechanism to ensure a consistent sample volume.

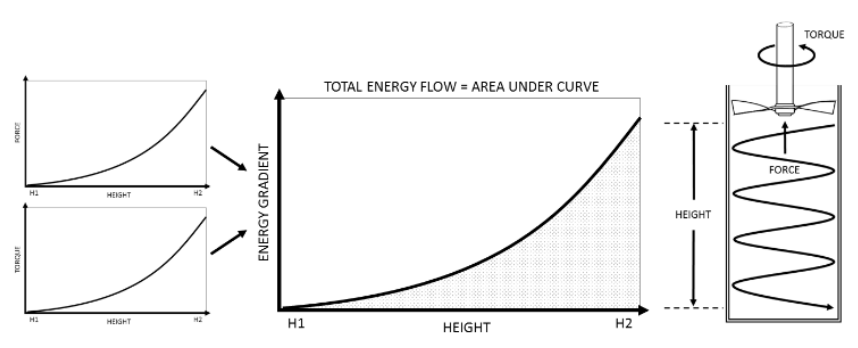

Fig. 1 Dynamic measurement principle

Other dynamic systems which measure the resistance to the passage of a blade, typically only measure axial force. This assumes that the powder bed acts as a continuous uniform body, whereas most powder beds contain air pockets and channels, especially more cohesive powders. As particles displaced by the blade fill these voids rather than colliding with and displacing surrounding particles, the energy is not fully transmitted to an axially positioned load cell [1]. Comparison of force and torque measurements demonstrates that the force is often an order of magnitude lower than torque. As such, not only are axial force measurements much less differentiating, but may also fail to correctly asses and rank more cohesive powders [1].

In addition to measuring the properties of the powder in a "poured" state, the addition of a controlled air feed allows the measurement of the flow energy as a function of air velocity. This is particularly useful for understanding the properties of powders in a low stress, aerated/fluidised state, for example during pneumatic conveying. Not all powders reach a fully fluidised state, especially cohesive or large/dense powders, this can be problematic for rheological techniques that require powders to be fluidisable.

At the opposite end of the spectrum, dynamic properties can be measured at higher stresses through application of an external force, such as compaction or vibration [5]. More recent advancements have also demonstrated the benefits of rheological measurements for quantifying caking behaviour in powders, especially for non-homogenous caking [6].

\section{Additional test methodologies}

Whilst not a direct measure of the powder flowability, the bulk properties of Compressibility and Permeability can have considerable influence on powder behaviour during 
unit operations. For example, permeability can affect flow from hoppers and silos as well as filling applications, and compressibility is relevant to storage or during compaction processes such as tabletting and roll compaction. Finally, shear properties are useful for understanding how powders transition from a high stress, static state to a dynamic state. Measurements of Wall Friction quantify the ease with which a powder will move against process equipment surfaces.

\section{Case studies}

In both case studies, the powders were characterised using the FT4 Powder Rheometer (Dynamic Flow, Bulk and Shear tests). The results were compared with known process behaviour in order to identify the most relevant parameters.

\subsection{Spray coating}

Three samples of a polymer powder were used in a spray coating application using a corona charging system. Sample A exhibited good performance in terms of flowing through the nozzle and adhering to the substrate, and Sample B showed acceptable behaviour, but Sample C was poor in both aspects; causing blockages in the nozzle and subsequently falling away from the substrate during transportation to the kiln. Both particle size analysis and shear testing failed to discriminate between the samples,

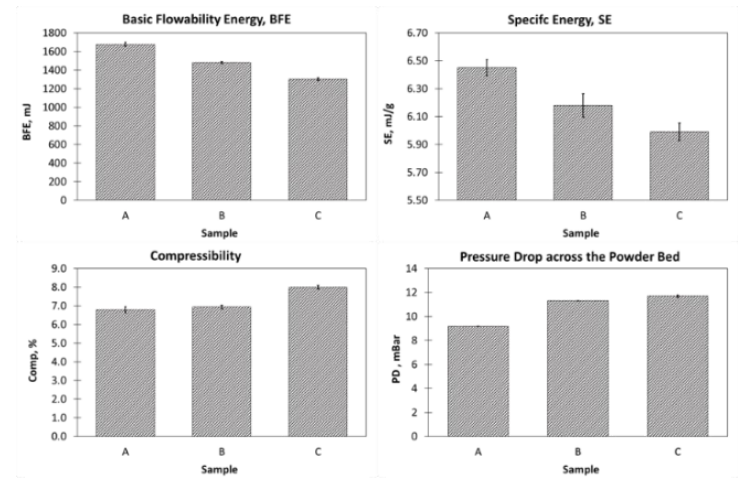

Fig. 2. Dynamic and Bulk results for polymers A to $\mathrm{C}$ used in a spray coating application.

Sample A generated the highest Basic Flowability Energy (BFE) and Specific Energy (SE) of the three samples (fig. 2), which together indicate greater cohesion and particle-particle interlocking. Sample $C$ generated the lowest BFE and SE, suggesting that a degree of interparticular cohesion is required to form a uniform coating on the substrate, and that Sample $\mathrm{C}$ does not meet this criterion.

Sample C was the most compressible of the samples, indicating a greater propensity to compact under forced flow conditions, such as when drawing the powder from the storage vessel into the fluidisation chamber. The greater propensity to compact will promote the formation of agglomerates, inhibiting both the spraying and charging operations in the nozzle.

Sample A generated the lowest Pressure Drop across the Powder Bed indicating that it is the most permeable. This suggests that it will be the most free-flowing under conveyance, and that once fluidised, is likely to flow more readily within an air stream. Sample C was least permeable, generating the highest Pressure Drop across the Powder Bed, which is likely to cause more erratic, pulsatile flow into the fluidisation chamber, and unstable flow of the fluidised mass.

The results suggest that a degree of cohesion is required to form a uniform coating but susceptibility to agglomeration and erratic flow is problematic to the process. Sample C, with the lowest BFE and Permeability, and the highest Compressibility, is most sensitive to compaction during conveyance to the fluidisation chamber, forming agglomerates that can block the nozzle and cause inconsistent charging. This also highlights a common misconception that the most free-flowing samples will provide the best process performance, with the least cohesive sample having the worst process performance in this example. Indeed, freeflowing powders have their own problems, such segregation and flooding.

\subsection{Capsule filling}

Three batches of a pharmaceutical blend were used to fill sachets for oral dosing. The three batches flowed differently from the filling shoe into the sachet, resulting in significant weight variation in the sachets. Sample A exhibited good performance and Sample B (with a wider particle size distribution) was classed as average. Sample $\mathrm{C}$ had the same particle size distribution as Sample A, but performed very poorly in the process, suggesting that particle size alone did not dictate performance. Samples where characterised using the FT4 Powder Rheometer.

Sample A generated the lowest BFE, requiring less energy to move the blade through the powder (fig 3). Sample C generated the highest. The low BFE is indicative of a powder that is able to flow more freely under the forced flow conditions present in a shoe feeder operation. Sample C generated the highest Pressure Drop across the Powder Bed (lowest permeability), indicating a greatest resistance to the passage of air. Low permeability means that air entrained in the powder when it enters the sachet cannot escape, which can lead to high weight variation across a manufacturing run. Sample A was less permeable than Sample B, suggesting that an extreme value for this parameter may contribute to sub-optimal performance in this specific unit operation. Other filling applications may be more sensitive to variations in the permeability and therefore necessitate different acceptance criteria[7].

Differences in the permeability may also explain the aeration behaviour. Sample A generated the highest Aerated Energy (AE). Highly permeable powders allow 
air to traverse the bed readily with little influence on its packing structure. In contrast, Sample $\mathrm{C}$ generated the lowest AE, likely as a result of its lower permeability. Furthermore, powders that are sensitive to aeration may also be more prone to segregation and dusting, which can both have a detrimental impact on content uniformity.
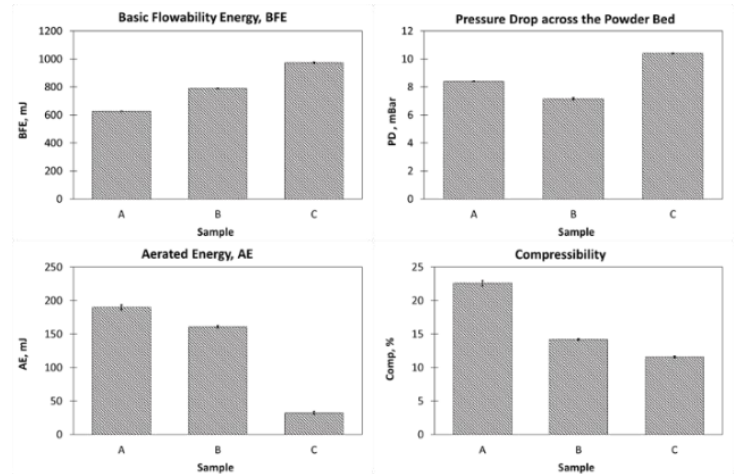

Fig. 3. Dynamic and Bulk results for pharmaceutical powders A to $\mathrm{C}$ used in a sachet filling application.

Sample A generated the highest Compressibility value, likely a result of the formation of agglomerates. however, this does not appear to have a negative impact on the filling operation, probably due to the low stress conditions imposed in the process demonstrating the importance of identifying test methodologies which represent process conditions.

The results show that powders with a low resistance to dynamic flow (low BFE), and a high permeability (high AE, lower Pressure Drop) perform best in this operation. In contrast, powders with a higher resistance to flow, and a greater sensitivity to aeration, perform poorly. This enables a design space to be defined, against which new formulations can be assessed to predict performance.

\section{Conclusion}

Both cases studies demonstrate that a single parameter may not be sufficient to fully rationalise the process performance, and that a multivariate approach is required. The case studies also demonstrate that the key properties are specific to each process; while BFE was an important parameter in understanding the process performance in both applications, the acceptance criteria were different. Expansion of the sample set would enable the upper and lower limits for each parameter to be more accurately defined, providing a more robust design window for each process or unit operation.

The spray coating application required a higher BFE, as degree of cohesion was necessary to ensure that the powders adhered to each other and formed a uniform coating, whereas higher cohesion was detrimental to the sachet filling application as it resulted in poor flow in the shoe feeder operation. A powder which works in the spray coating application is likely to perform badly in the sachet filling application. As such, it is necessary to understand the specific requirements/constraints for a given unit operation, furthermore it cannot be automatically assumed that the most free-flowing powder/granular system is the most suitable

Powder flowability is not an inherent material property, but is more about the ability of powder to flow in a desired manner in a specific piece of equipment. Successful processing demands that powders and processes are well-matched and it is not uncommon for the same powder to perform well in one process but poorly in another. This means that several characterisation methodologies are required, the results from which can be correlated with process ranking to produce a design space of parameters that correspond to acceptable process behaviour. Rather than relying on single number characterisation to describe behaviour across all processes, the multivariate approach of powder rheology simulates a range of unit operations, allowing for the direct investigation of a powder's response to various process and environmental conditions.

\section{References}

1. K. Brockbank, Novel Approaches to the Assessment of Pharmaceutical Powder Flow Behaviour, in School of Life Sciences. University of Bradford: Bradford, UK, (2011).

2. C. Ghoroi, L. Gurumurthy, D. J. Mcdaniel, L. J. Jallo, R. N. Davé, Powder Technology. 236, 63(2013).

3. D. Am Ende, M. Birch, S. J. Brenek, M. T. Maloney, Organic Process Research \& Development. 17, 1345(2013).

4. Z. Yan, S. A. Wilkinson, E. H. Turnbull, E. H. Stitt, M. M, Parametric Evalution for Powder Flowability using a Freeman Rheometer: A Discrete Element Method Study, in IV International Conference on Particle-based Methods - Fundamentals and Applications. CIMNE: Barcelona, Spain, (2015).

5. R. Freeman, Powder Technology. 174, 25(2007).

6. T. Freeman, K. Brockbank, B. Armstrong, Procedia Engineering. 102, 35(2015).

7. T. Freeman, J. Clayton, B. Armstrong, X. Fu, The Influence of Powder Flow Properties on the Filling of Dies and Capsules, in Particulate Systems Analysis 2011: Edinburgh, UK, (2011). 